\title{
Second-harmonic generation and self-phase modulation of few-cycle mid-infrared pulses
}

\author{
Bo-Han Chen, ${ }^{1,2}$ (1) Christina Hofer, ${ }^{1,2}$ loachim Pupeza, ${ }^{1,2}$ (1) and Peter Baum ${ }^{1,2,3, *}$ (1) \\ ${ }^{1}$ Ludwig-Maximilians-Universität München, Am Coulombwall 1, 85748 Garching, Germany \\ ${ }^{2}$ Max-Planck-Institute of Quantum Optics, Hans-Kopfermann-Str. 1, 85748 Garching, Germany \\ ${ }^{3}$ University of Konstanz, Universitätsstraße 10, 78457 Konstanz, Germany \\ *Corresponding author: peter.baum@uni-konstanz.de
}

Received 2 July 2019; revised 24 July 2019; accepted 25 July 2019; posted 25 July 2019 (Doc. ID 371040); published 14 August 2019

\begin{abstract}
Near-single-cycle mid-infrared pulses with a spectrum covering 5.4-11 $\mu \mathrm{m}$ are efficiently frequency-doubled in different GaSe crystals. The second-harmonic spectrum spans $3-4.3 \mu \mathrm{m}$ at a power conversion efficiency of $>20 \%$. We measure an effective nonlinear coefficient of $d_{\text {eff }} \approx 35 \mathrm{pm} / \mathrm{V}$. We also report on self-phase modulation and spectral broadening of the mid-infrared pulses in various bulk materials and find an increase of $45 \%$ of spectral width for $5 \mathrm{~mm}$ of Ge. These results demonstrate that nonlinear optical conversions can efficiently be driven by few-cycle mid-infrared radiation. () 2019 Optical Society of America
\end{abstract}

https://doi.org/10.1364/OL.44.004079

Provided under the terms of the OSA Open Access Publishing Agreement

The outstanding coherence of laser radiation affords an exceptional control over the electric field of light at the level of the optical cycles. Of particular interest are waveform-controlled, few-cycle pulses with field strengths on the order of the atomic Coulomb field, $\sim 0.1 \mathrm{~V} / \AA ̊$. Such pulses enable the study of quasiparticles and nonperturbative nonlinear optics in crystals [1-3] or gases [4-6], the all-optical control of electron beams [7-10], or field-resolved spectroscopy of molecular vibrations [11-13]. However, mid-infrared laser sources often do not operate at appropriate central wavelengths or enough coherent spectral width for such experiments. In particular, realizing waveform-controlled single-cycle pulses with a simultaneous spectral coverage below and above $\sim 5 \mu \mathrm{m}$, the transparency threshold of oxide crystals [14] remains a challenge.

In this Letter, we report efficient second-harmonic generation (SHG) and self-phase modulation (SPM) of few-cycle pulses in the long-wave mid-infrared region of the optical spectrum around $5-11 \mu \mathrm{m}$. These results advance earlier findings made with picosecond pulses from free-electron lasers [15] or $\mathrm{CO}_{2}$ lasers $[16,17]$ into the regime of broadband waveformcontrolled pulses from femtosecond lasers and demonstrate the feasibility of few-cycle nonlinear optical conversions with mid-infrared radiation. The SPM results expand earlier findings with supercontinuum generation $[18,19]$ to the regime of simple and direct spectral broadening without filament formation and cascaded nonlinear optics.
The mid-infrared pulses used for our experiments are obtained from a non-collinear optical parametric amplifier composing white-light seed generation and broadband parametric amplification of the near-infrared part around $1200 \mathrm{~nm}$ followed by type-I difference frequency mixing with the $1030 \mathrm{~nm}$ pulses from the driving laser (Pharos PH1-20, Light Conversion) [20]. The output mid-infrared pulses have a pulse energy of $400 \mathrm{~nJ}$ at a repetition rate of $50 \mathrm{kHz}$ and a central wavelength of $7 \mu \mathrm{m}$. The spectrum covers $5.4-11 \mu \mathrm{m}$ $(27.3-55.5 \mathrm{THz})$ at $-20 \mathrm{~dB}$ intensity, and the ultrashort electric field has a pulse duration of $\sim 34$ fs or $\sim 1.4$ optical cycles (intensity full width at half-maximum [FWHM]). Figure 1(a) shows an electro-optic sampling measurement, made with a $20 \mu \mathrm{m}$ thick GaSe crystal and $12 \mathrm{fs}$ visible probe pulses from a supercontinuum source [20] of the electric field, as it enters our optical setup.

Figure 1(b) depicts the experiment. The few-cycle mid-infrared pulses are focused and re-collimated by off-axis parabolic mirrors ( $f=50.8 \mathrm{~mm}$, MPD129-M01, Thorlabs) and then sent to diagnostics. In the focus, we measure a beam diameter of $\sim 84 \mu \mathrm{m}\left(1 / e^{2}\right.$ of the intensity), measured with a $\mathrm{Si}$ microbolometer array (WinCamD-FIR2-16-HR, Dataray). Therefore, the pulses have a peak intensity of $\sim 400 \mathrm{GW} / \mathrm{cm}^{2}$ and a peak field strength of $\sim 1.7 \mathrm{GV} / \mathrm{m}$ or $0.17 \mathrm{~V} / \AA$. The output spectra after the nonlinear optical interaction are measured with a Fourier-transform infrared spectrometer (L-FTS, LASNIX), and the power is detected with a thermopile

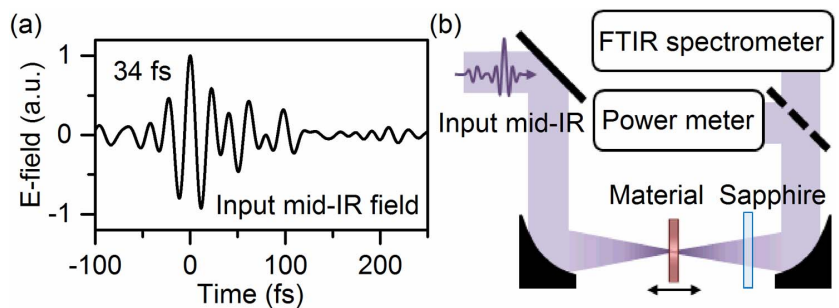

Fig. 1. Fundamental mid-infrared pulses and the experimental setup. (a) Electric field measured with electro-optic sampling and retrieved from deconvolution with the detection's response function. (b) Experimental setup. FTIR, Fourier-transform infrared spectrometer; dashed line, flip mirror. 
absorption sensor (PS19, Coherent). A sapphire filter is used in some of our experiments to filter out unwanted spectral components.

In a first experiment, we report on SHG. We choose GaSe as the nonlinear crystal, because of its rather high optical nonlinearity of $\sim 50 \mathrm{pm} / \mathrm{V}[21]$ and its broadband transparency range $(0.7-18 \mu \mathrm{m})[22,23]$. We investigate two different crystal thicknesses, 250 and $465 \mu \mathrm{m}$, measured by optical interferometry and femtosecond group delay measurements. The crystal main axis is tilted by $\sim 33^{\circ}$ with respect to the optical axis in order to satisfy type-I phase matching at an internal angle of $11^{\circ}$ after refraction. In this geometry, the temporal walk-off is $10 \mathrm{fs}$ for the $250 \mu \mathrm{m} \mathrm{GaSe}$ and 20 fs for the $465 \mu \mathrm{m} \mathrm{GaSe}$, and the spatial walk-off is $54 \mathrm{mrad}$. The crystal is moved along the beam propagation direction in order to maximize the second-harmonic output power. A $1 \mathrm{~mm}$ sapphire plate filters away the remaining input mid-infrared radiation. The second-harmonic pulses are then sent to diagnostics.

Figure 2 depicts the results. The input spectrum to be frequency-doubled is shown in Fig. 2(a), covering the spectral range between $5.4-11 \mu \mathrm{m}$ at $-20 \mathrm{~dB}$ intensity. The dashed line shows the calculated phase-matching function $|\operatorname{sinc}(\Delta k / 2)|^{2}$ for $250 \mu \mathrm{m}$ of $\mathrm{GaSe}$ at the experimental tilt angle. In Figure 2(b), the dotted curve, shows the corresponding second-harmonic spectrum that could be expected from a thin $(250 \mu \mathrm{m}) \mathrm{GaSe}$ crystal. However, the electro-optic sampling data of Fig. 1(a) reveal some residual second-order dispersion of $\sim-100 \mathrm{fs}^{2}$ and a substantial third-order dispersion of $\sim 15700 \mathrm{fs}^{3}$ of the input pulses and, therefore, a non-Gaussian shape in time. In the frequency domain, components at the (a)

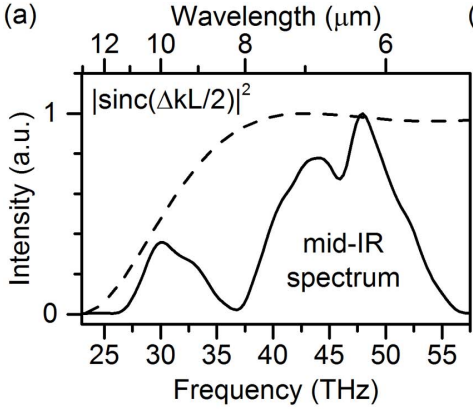

(b)

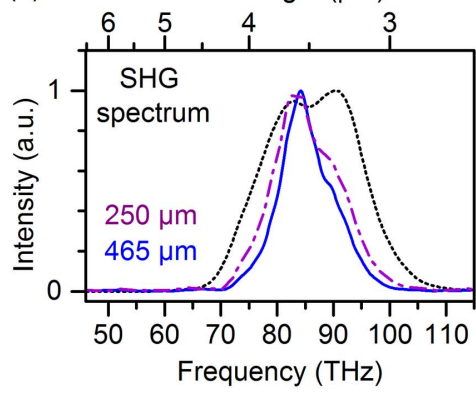

(c)

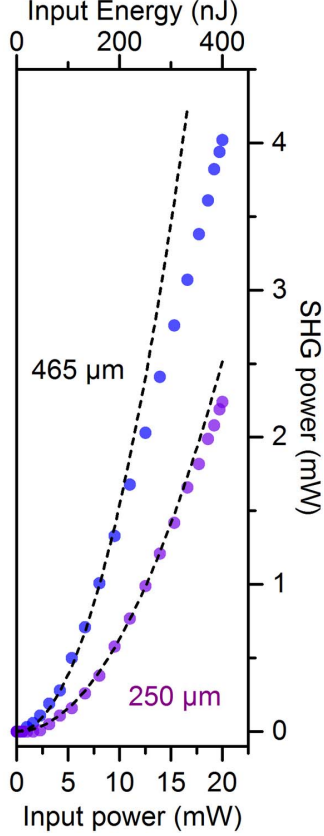

Fig. 2. SHG. (a) Fundamental mid-infrared spectrum (solid) and phase-matching function $|\operatorname{sinc}(\Delta k / 2)|^{2}$ (dashed) of the $250 \mu \mathrm{m}$ GaSe crystal. (b) Second-harmonic spectrum generated from $250 \mu \mathrm{m}$ of GaSe (violet, dotted-dashed) and $465 \mu \mathrm{m}$ GaSe (blue, solid). Black-dotted line: simulated second-harmonic spectrum according to (a) for the $250 \mu \mathrm{m}$ crystal. (c) Power dependence measurement. Scattered dots, measured data; dashed lines, quadratic fits. wings of the input spectrum are less well compressed than the central part. Pulses with third-order dispersion, therefore, have a narrower second-harmonic spectrum than expected from the phase-matching limit [24].

The blue solid line shows the measured SHG spectrum for the $465 \mu \mathrm{m}$ GaSe crystal and the violet dashed-dotted line shows the measured spectrum for the $250 \mu \mathrm{m}$ crystal. Both spectra, indeed, are somewhat narrower than the expectation for non-chirped input pulses, but the spectral bandwidth at $-20 \mathrm{db}$ is still $3-4.3 \mu \mathrm{m}$ for the $465 \mu \mathrm{m}$ crystal and $2.8-4.4 \mu \mathrm{m}$ for the $250 \mu \mathrm{m}$ crystal. A further improvement is expected from controlling the higher-order dispersion, for example, with state-of-the-art infrared multilayer optics [25].

Figure 2(c) shows the output power as a function of input power (pulse energy). For both the $465 \mu \mathrm{m} \mathrm{GaSe} \mathrm{crystal} \mathrm{(blue}$ dots) and the $250 \mu \mathrm{m}$ crystal (violet dots), we see a characteristic quadratic increase with pump power. Due to walk-off effects, back-conversion, third-order nonlinearities or multiphoton absorption by impurities or dislocations, saturation effects become observable above $15 \mathrm{~mW}$ of pump power for the $465 \mu \mathrm{m}$ crystal and above $20 \mathrm{~mW}$ for the $250 \mu \mathrm{m}$ crystal. A maximum output power of $4 \mathrm{~mW}$ was achieved with the $465 \mu \mathrm{m}$ crystal after the sapphire filter. This value corresponds to a pulse energy of $80 \mathrm{~nJ}$, a power conversion efficiency of $20 \%$, and a quantum efficiency of $10 \%$, from before the GaSe to after all filters of the experiment.

In order to determine the absolute conversion efficiency inside the GaSe crystal, we consider the Fresnel losses of the input mid-infrared pulses at the crystal's front surface (reflectivity $\sim 21 \%$ at $7 \mu \mathrm{m}$ wavelength) and the losses of the secondharmonic pulses at the back surface (reflectivity $\sim 17 \%$ at $3.5 \mu \mathrm{m}$ wavelength). The sapphire filter has a total transmission of $\sim 87 \%$ at $3.5 \mu \mathrm{m}$ wavelength. In principle, all these losses can be avoided by using proper anti-reflection coatings, but those were not at our disposal at the time of the experiment. If avoiding the interface losses, the second-harmonic power with the $465 \mu \mathrm{m}$ crystal would be $5.5 \mathrm{~mW}$, the power conversion efficiency would be $35 \%$, and the quantum efficiency would be $18 \%$. These values allow us to estimate the effective nonlinear coefficient $d_{\text {eff }}$ in our experiment. Taking into account the beam size at the focus, the phase-matching condition, and the crystal length, we obtain $\sim 35 \mathrm{pm} / \mathrm{V}$, with an estimated error of $\pm 20 \%$. This result is a lower limit due to the potential contributions of temporal and spatial walk-off. The measured $d_{\text {eff }}$ compares to previous results obtained with nanosecond [26] and sub-nanosecond [21] lasers and shows that GaSe maintains its beneficial nonlinearity when entering the fewcycle regime of pulses with close to octave-broad spectra.

Figure 3(a) shows the far-field beam profile of the incoming mid-infrared pulses, measured with a pyroelectric array camera (Pyrocam IIIHR, Spiricon, Ophir Photonics). Figures 3(b) and 3 (c) show the beam profiles of the second-harmonic pulses from the 250 and $465 \mu \mathrm{m} \mathrm{GaSe}$ crystals after the sapphire filter and beam collimation. We see that the second-harmonic beam sizes are smaller than the input beam size by a factor of about $\sqrt{2}$, as expected. The slight asymmetry in Fig. 3(c) is probably caused by spatial walk-off ( $54 \mathrm{mrad})$ or the onset of cascaded processes in the thicker crystal.

The fundamental mid-infrared pulses driving our experiments have a passively stable carrier-envelope phase (CEP) [20] and the second-harmonic pulses should therefore also 


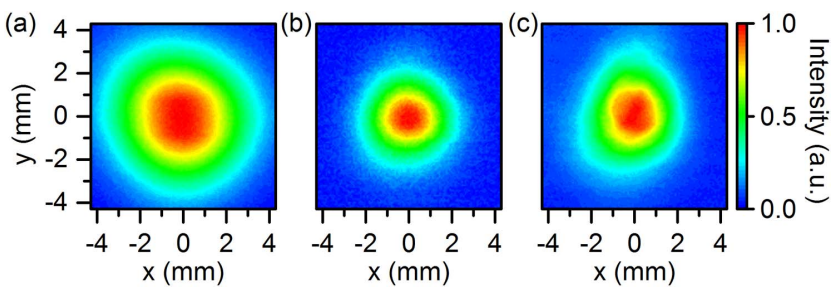

Fig. 3. Far-field beam profiles. (a) Input mid-infrared beam. (b) Second-harmonic beam from $250 \mu \mathrm{m}$ of GaSe. (c) Secondharmonic beam from $465 \mu \mathrm{m}$ of GaSe.

be CEP-stable. The fundamental and second-harmonic pulses are therefore suitable for coherent short-pulse synthesis [27,28]. Figure 4 shows the calculated Fourier-limited pulse shape and electric field when assuming a combination of the measured fundamental spectrum with the measured SHG output $(465 \mu \mathrm{m}$ crystal) at the measured power ratio of roughly $5: 1$. We suppose equal polarization and CEP to produce cosine-like pulses. The relative delay is assumed to be zero $[27,28]$. The result, plotted in Fig. 4(b) is an electric field with merely 0.7 optical cycles within a FWHM pulse duration of $12 \mathrm{fs}$. With a hypothetical $2 \lambda$ focus diameter, the peak electric field could reach $>10 \mathrm{GV} / \mathrm{m}$ or $1 \mathrm{~V} / \AA ̊$. The central peak's electric field is $>2.3$ times stronger than any neighboring optical cycles. Such an ultrashort synthesized light field, obtainable with one single and simple SHG stage, could probably be useful for high-field physics or ultrabroadband spectroscopy.

In a second experiment, we report SPM and spectral broadening of our mid-infrared few-cycle pulses in different bulk materials $[18,19]$. In contrast to fibers [29], bulk materials are better scalable to higher pulse energies and average powers. The setup is similar as above but now without the sapphire filter. The parabolic mirrors have a slightly longer focal length ( $f=101.6 \mathrm{~mm}$, MPD149-M01, Thorlabs), producing a focus with a diameter of $175 \mu \mathrm{m}\left(1 / e^{2}\right.$ intensity) and a peak intensity of $92 \mathrm{GW} / \mathrm{cm}^{2}$. The investigated bulk materials are $5 \mathrm{~mm}$ of $\mathrm{KCl}, 5 \mathrm{~mm}$ of $\mathrm{CaF}_{2}, 2 \mathrm{~mm}$ of $\mathrm{KBr}, 5 \mathrm{~mm}$ of $\mathrm{ZnSe}$ and $5 \mathrm{~mm}$ of $\mathrm{Ge}$, each placed into the beam under normal incidence.

Figure 5 depicts the results. For $\mathrm{KCl}, \mathrm{CaF}_{2}$ and $\mathrm{KBr}$ we find no substantial effects at the given peak intensity. Figure 5(a) shows the results for $\mathrm{ZnSe}$ when moving the crystal through the focus position. Figure 5(b) shows the results for Ge. The change of the Fourier-limited pulse duration is plotted

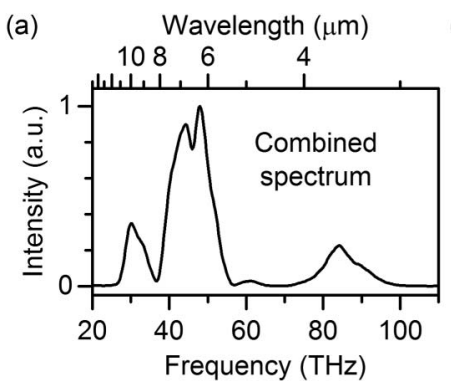

(b)

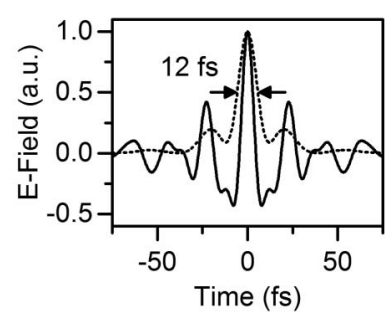

Fig. 4. Hypothetical waveform synthesizer. (a) Combined spectrum of the fundamental mid-infrared and second-harmonic pulses. (b) Fourier-limited pulse shape (dotted) and electric field for a cosine-like pulse (solid).
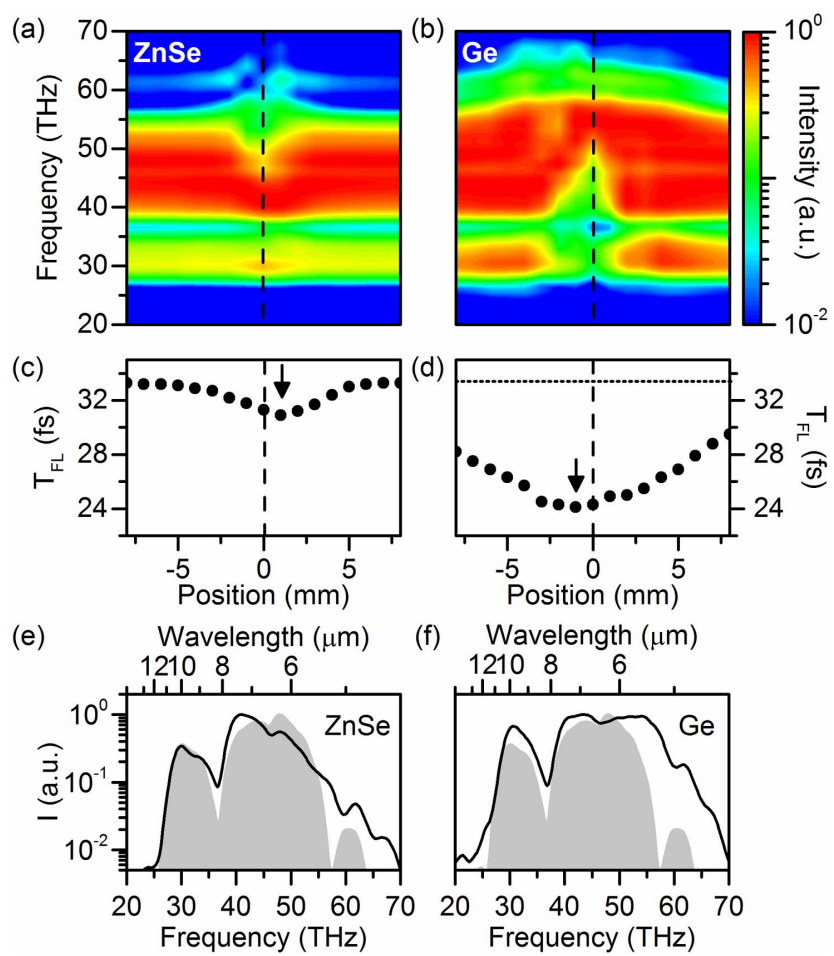

Fig. 5. SPM and spectral broadening. (a) Output spectrum as a function of the crystal position for $\mathrm{ZnSe}$. (b) Output spectrum for Ge. (c) Fourier-limited pulse duration $T_{\mathrm{FL}}$ for $\mathrm{ZnSe}$ as a function of crystal position. (d) Fourier-limited pulse duration $T_{\mathrm{FL}}$ for Ge. The arrows mark the best broadening for the shortest pulses. (e) Broadest spectrum achieved with $\mathrm{ZnSe}$. (f) Broadest spectrum achieved with Ge. Gray area shows the original mid-infrared spectrum for comparison. I, normalized spectral intensity.

for the two crystals in Figs. 5(c) and 5(d). From the spectra in Figs. 5(a) and 5(b) we see in both cases first an increase and afterwards a decrease of the broadening when moving the crystal through the focus. Interestingly, the spectral shapes are not symmetric with respect to the focus position and the most broadband spectrum is seen slightly after the focus for $\mathrm{ZnSe}$ and slightly before the focus for Ge. Both crystals are thick enough that spectral broadening, chirp and intensity changes occur simultaneously and affect each other [30]. The fact that the broadest spectrum is observed slightly after the focus for $\mathrm{ZnSe}$ and slightly before the focus for $\mathrm{Ge}$ is probably related to the different signs of first-order dispersion in these two materials $\left(\mathrm{ZnSe}\right.$, about $-406 \mathrm{fs}^{2}$; Ge, about $\left.+567 \mathrm{fs}^{2}\right)[31,32]$.

For $\mathrm{ZnSe}$, the best broadened mid-infrared spectrum, plotted in Fig. 5(e), supports a Fourier-limited pulse duration of 30.7 fs. For Ge, the best broadened spectrum is plotted in Fig. 5(f) and shows a Fourier limit of 24.8 fs. This value corresponds to a shortening of $27 \%$. The spectral width at $-20 \mathrm{~dB}$ intensity increases by $45 \%$ and now covers $27-68 \mathrm{THz}$ in a coherent way. SPM causes mostly a smooth, quadratic spectral phase and compression to these Fourier limits, therefore, should be realistic with bulk materials, for example, GaAs [19], or with chirped mid-infrared mirrors [33].

A somewhat detrimental effect of $\mathrm{Ge}$ is a substantial drop of the mid-infrared transmission to $\sim 11 \%$ at maximum 
broadening. This loss is probably associated with multi-photon absorption owning to Ge's low bandgap of $0.66 \mathrm{eV}$ [34]. However, no long-term damage was observed, and the spectrally broadened output pulses still have $44 \mathrm{~nJ}$ of pulse energy and an average power of $2.2 \mathrm{~mW}$, making them useful for spectroscopy or further nonlinear conversions.

In summary, the reported two results on SHG and SPM show that nonlinear optics with driving pulses in the long-wave midinfrared region of the spectrum is feasible, easy, and efficient. The SHG spectrum obtained at the reported conditions covers an interesting spectral region for applications in molecular spectroscopy. In particular, the spectral coverage of high-sensitivity, highpower, field-resolved spectroscopy of biofluids [12] could be extended from the spectral regions of nucleic acids and carbohydrates to those of proteins and lipids [35]. The ability to cover novel spectral ranges in new ways with modern mid-infrared lasers will potentially also open up advanced possibilities in electron-beam control [7-10], sub-cycle electron microscopy [36], or high-field attosecond science [1-4,37,38].

Funding. H2020 European Research Council (647771); Munich-Centre for Advanced Photonics (MAP).

\section{REFERENCES}

1. O. Schubert, M. Hohenleutner, F. Langer, B. Urbanek, C. Lange, U. Huttner, D. Golde, T. Meier, M. Kira, S. W. Koch, and R. Huber, Nat. Photonics 8, 119 (2014)

2. F. Langer, M. Hohenleutner, C. P. Schmid, C. Poellmann, P. Nagler, T. Korn, C. Schüller, M. S. Sherwin, U. Huttner, J. T. Steiner, S. W. Koch, M. Kira, and R. Huber, Nature 533, 225 (2016).

3. M. T. Hassan, T. T. Luu, A. Moulet, O. Raskazovskaya, P. Zhokhov, M. Garg, N. Karpowicz, A. M. Zheltikov, V. Pervak, F. Krausz, and E. Goulielmakis, Nature 530, 66 (2016).

4. T. Popmintchev, M. C. Chen, D. Popmintchev, P. Arpin, S. Brown, S. Alisauskas, G. Andriukaitis, T. Balciunas, O. D. Mucke, A. Pugzlys, A. Baltuska, B. Shim, S. E. Schrauth, A. Gaeta, C. Hernandez-Garcia, L. Plaja, A. Becker, A. Jaron-Becker, M. M. Murnane, and H. C. Kapteyn, Science 336, 1287 (2012).

5. B. Bergues, D. E. Rivas, M. Weidman, A. A. Muschet, W. Helml, A. Guggenmos, V. Pervak, U. Kleineberg, G. Marcus, R. Kienberger, D. Charalambidis, P. Tzallas, H. Schröder, F. Krausz, and L. Veisz, Optica 5, 237 (2018).

6. D. J. Wilson, A. M. Summers, S. Zigo, B. Davis, S. Robatjazi, J. A. Powell, D. Rolles, A. Rudenko, and C. A. Trallero-Herrero, Sci. Rep. 9, 6002 (2019).

7. C. Kealhofer, W. Schneider, D. Ehberger, A. Ryabov, F. Krausz, and P. Baum, Science 352, 429 (2016).

8. D. F. Zhang, A. Fallahi, M. Hemmer, X. J. Wu, M. Fakhari, Y. Hua, H. Cankaya, A. L. Calendron, L. E. Zapata, N. H. Matlis, and F. X. Kärtner, Nat. Photonics 12, 336 (2018).

9. D. Ehberger, A. Ryabov, and P. Baum, Phys. Rev. Lett. 121, 094801 (2018).

10. P. Yousefi, N. Schönenberger, J. Mcneur, M. Kozák, U. Niedermayer, and P. Hommelhoff, Opt. Lett. 44, 1520 (2019).

11. I. Pupeza, D. Sanchez, J. Zhang, N. Lilienfein, M. Seidel, N. Karpowicz, T. Paasch-Colberg, I. Znakovskaya, M. Pescher,
W. Schweinberger, V. Pervak, E. Fill, O. Pronin, Z. Wei, F. Krausz, A. Apolonski, and J. Biegert, Nat. Photonics 9, 721 (2015).

12. I. Pupeza, M. Huber, W. Schweinberger, M. Trubetskov, S. A. Hussain, L. Vamos, O. Pronin, F. Habel, V. Pervak, N. Karpowicz, E. Fill, A. Apolonski, M. Zigman, A. M. Azzeer, and F. Krausz, in Conference on Lasers and Electro-Optics Europe \& European Quantum Electronics Conference (CLEO/Europe-EQEC) (2017), pp. 1.

13. A. S. Kowligy, H. Timmers, A. Lind, U. Elu, F. C. Cruz, P. G. Schunemann, J. Biegert, and S. A. Diddams, Sci. Adv.5, eaaw8794 (2019).

14. V. Petrov, Opt. Mater. 34, 536 (2012)

15. J. M. Auerhammer and E. R. Eliel, Opt. Lett. 21, 773 (1996).

16. P. B. Corkum, P. P. Ho, R. R. Alfano, and J. T. Manassah, Opt. Lett. 10, 624 (1985).

17. J. J. Pigeon, S. Ya. Tochitsky, C. Gong, and C. Joshi, Opt. Lett. 39, 3246 (2014).

18. A. A. Lanin, A. A. Voronin, E. A. Stepanov, A. B. Fedotov, and A. M. Zheltikov, Opt. Lett. 39, 6430 (2014).

19. A. A. Lanin, A. A. Voronin, E. A. Stepanov, A. B. Fedotov, and A. M. Zheltikov, Opt. Lett. 40, 974 (2015).

20. B. Chen, E. Wittmann, Y. Morimoto, E. Riedle, and P. Baum, Opt Express 27, 21306 (2019).

21. K. L. Vodopyanov, J. Opt. Soc. Am. B 10, 1723 (1993).

22. K. R. Allakhverdiev, M. Ö. Yetis, S. Özbek, T. K. Baykara, and E. Y. Salaev, Laser Phys. 19, 1092 (2009).

23. J. Guo, J. J. Xie, D. A. J. Li, G. L. Yang, F. Chen, C. R. Wang, L. M. Zhang, Y. M. Andreev, K. A. Kokh, G. V. Lanskii, and V. A. Svetlichnyi, Light Sci. Appl. 4, e362 (2015).

24. P. Baum, S. Lochbrunner, and E. Riedle, Appl. Phys. B 79, 1027 (2004).

25. F. Habel, M. Trubetskov, and V. Pervak, Opt. Express 24, 16705 (2016).

26. N. B. Singh, D. R. Suhre, V. Balakrishna, M. Marable, R. Meyer, N. Fernelius, F. K. Hopkins, and D. Zelmon, Prog. Cryst. Growth Charact. Mater. 37, 47 (1998).

27. C. Schmidt, J. Bühler, B. Mayer, A. Pashkin, A. Leitenstorfer, and D. V. Seletskiy, J. Opt. 18, 05LT01 (2016).

28. J. Bühler, C. Schmidt, H. Schneider, M. Helm, A. Pashkin, and D. V. Seletskiy, J. Infrared, Millimeter, Terahertz Waves 38, 808 (2017).

29. C. R. Petersen, U. Moller, I. Kubat, B. B. Zhou, S. Dupont, J. Ramsay, T. Benson, S. Sujecki, N. Abdel-Moneim, Z. Q. Tang, D. Furniss, A. Seddon, and O. Bang, Nat. Photonics 8, 830 (2014).

30. B. Chen, T. Nagy, and P. Baum, Opt. Lett. 43, 2876 (2018).

31. H. W. Icenogle, B. C. Platt, and W. L. Wolfe, Appl. Opt. 15, 2348 (1976).

32. B. Tatian, Appl. Opt. 23, 4477 (1984).

33. F. Habel and V. Pervak, Appl. Opt. 56, C71 (2017).

34. P. J. Collings, Am. J. Phys. 48, 197 (1980).

35. M. J. Baker, J. Trevisan, P. Bassan, R. Bhargava, H. J. Butler, K. M. Dorling, P. R. Fielden, S. W. Fogarty, N. J. Fullwood, K. A. Heys, C. Hughes, P. Lasch, P. L. Martin-Hirsch, B. Obinaju, G. D. Sockalingum, J. Sulé-Suso, R. J. Strong, M. J. Walsh, B. R. Wood, P. Gardner, and F. L. Martin, Nat. Protoc. 9, 1771 (2014).

36. A. Ryabov and P. Baum, Science 353, 374 (2016).

37. V. S. Yakovlev, M. I. Stockman, F. Krausz, and P. Baum, Sci. Rep. 5, 14581 (2015).

38. G. J. Stein, P. D. Keathley, P. Krogen, H. K. Liang, J. P. Siqueira, C. L. Chang, C. J. Lai, K. H. Hong, G. M. Laurent, and F. X. Kartner, J. Phys. B 49, 155601 (2016) 